\title{
Effects of quetiapine on sleep architecture in patients with unipolar or bipolar depression
}

This article was published in the following Dove Press journal:

Neuropsychiatric Disease and Treatment

6 August 2010

Number of times this article has been viewed

Laura Gedge'

Lauren Lazowski'

David Murray ${ }^{2}$

Ruzica Jokic ${ }^{2,3}$

Roumen Milev 2,3

'Centre for Neuroscience Studies, 2Department of Psychiatry, Queen's University, Kingston, ${ }^{3}$ Providence

Care-Mental Health Services,

Kingston, Ontario, Canada
Correspondence: Roumen Milev Department of Psychiatry, Queen's University, Providence Care-Mental Health Services, 752 King Street West, Postal Bag 603, Kingston, Ontario, Canada, K7L 4X3

Tel + I 6I35485567 ext 5823

Fax + I 6I35485580

Email roumen.milev@queensu.ca
Objective: To determine the effect of adjunctive quetiapine therapy on the sleep architecture of patients with bipolar or unipolar depression.

Methods: This is a prospective, single-blind, repeated measures polysomnographic study. Sleep architecture was analyzed by overnight polysomnography, and subjective sleep quality was measured using the Pittsburgh Sleep Quality Index. The Hamilton Rating Scale for Depression, Montgomery Asberg Depression Rating Scale, Young Mania Rating Scale, and Clinical Global Impression-Severity Scale were employed to quantify changes in illness severity with adjunctive quetiapine treatment. Polysomnographs and clinical measures were administered at baseline, after 2-4 days of treatment, and after 21-28 days of quetiapine treatment. The average dose of quetiapine was $155 \mathrm{mg}$, ranging from $100-200 \mathrm{mg}$.

Results: Adjunctive quetiapine therapy did not significantly alter sleep efficiency, sleep continuity, or Pittsburgh Sleep Quality Index scores. Respiratory Disturbance Index and percentage of total time in rapid eye movement (REM) sleep significantly decreased and the percentage of total time in non-REM sleep, and duration of Stage 2 and non-REM sleep significantly increased after 2-4 days of quetiapine treatment. Illness severity significantly decreased over time.

Conclusions: Adjunctive quetiapine treatment alters sleep architecture in patients with major depressive disorder or bipolar disorder, which may partially explain its early antidepressant properties. Changes in sleep architecture are more robust and significant within two to four days of starting treatment.

Keywords: quetiapine, sleep architecture, depression, bipolar disorder

\section{Introduction}

Quetiapine is an atypical antipsychotic agent and is approved by the United States Food and Drug Administration to treat schizophrenia, major depressive disorder (MDD) and both manic and depressive episodes associated with bipolar disorder. The drug acts as an antagonist at serotonin $\left(5-\mathrm{HT}_{1 \mathrm{~A}}\right.$ and $\left.5-\mathrm{HT}_{2}\right)$, dopamine $\left(\mathrm{D}_{1}\right.$ and $\left.\mathrm{D}_{2}\right)$, histamine $\left(\mathrm{H}_{1}\right)$, and adrenergic $\alpha 1$ and $\alpha 2$ receptors. ${ }^{1}$ Results from the BOLDER I and II studies, which were two large, multicenter, randomized, placebo-controlled trials, show that quetiapine is efficacious in the treatment of acute bipolar depression as monotherapy. ${ }^{2,3}$ These studies led to guidelines recommending quetiapine for use as first-line monotherapy treatment for bipolar depression. ${ }^{4}$ Use of an atypical antipsychotic as an adjuvant therapy with selective serotonin reuptake inhibitors (SSRIs) has shown benefits in large trials of olanzapine use with fluoxetine. ${ }^{5,6}$ The use of quetiapine as an adjunctive therapy has been shown to be beneficial in smaller, open-label studies of patients with bipolar depressive episodes. ${ }^{7,8}$ Large, randomized placebo-controlled

submit your manuscript $\mid$ www.dovepress.com 
trials have found the use of quetiapine as monotherapy, or in combination with another antidepressant, to be efficacious in the treatment of MDD. ${ }^{9-11}$

Patients with MDD and bipolar disorder frequently experience sleep disturbances. ${ }^{12}$ Poor sleep quality and/or quantity are observed in up to $90 \%$ of depressed patients. ${ }^{13}$ Insomnia is a risk factor for development of major depressive episodes and may precede the onset of depression in those with recurrent illness. ${ }^{14-16}$ Sleep disturbance is also a risk factor for suicide. ${ }^{17}$ Studies of sleep architecture using polysomnography (PSG) have demonstrated that sleep in depressed patients tends to be characterized by decreased sleep efficiency, a reduction of slow-wave sleep (SWS), and a disinhibition of rapid eye movement (REM) sleep, manifested by a shortening of REM sleep latency, an increase in REM length, and an increased REM density. ${ }^{18,19}$

Low doses of quetiapine alter the sleep architecture of healthy individuals. ${ }^{20,21}$ Somnolence is a side effect of quetiapine treatment, leading to prescription in an off-label fashion when this side effect is desired. ${ }^{22}$ The somnolence effect of quetiapine is thought to arise from its $5-\mathrm{HT}_{2}$ and $\mathrm{H}_{1}$ receptor blockade capabilities, similar to those of medications used as sedatives, including the antidepressants mirtazapine, trazodone, and trimipramine. Qualitative sleep studies using the Pittsburgh Sleep Quality Index (PSQI) have shown improvements in the sleep quality of depressed patients after initiation of quetiapine therapy. ${ }^{23,24}$

The effect of quetiapine on sleep architecture, measured by PSG, has not been studied in patients experiencing a major depressive episode. Clinical practice indicates that quetiapine has sedating properties, and its sedative effects may play an important role in restoring quality of sleep in patients with MDD or bipolar disorder who frequently experience sleep disturbances as part of their illness. We hypothesize that quetiapine alters the subjective sleep quality and PSG sleep architecture of patients with MDD or bipolar disorder. We expect that quetiapine will improve sleep efficiency, decrease sleep latency and duration of REM sleep, and increase the duration of SWS. If such changes occur and can be related to the improvement of depressive symptomatology, it is suggested that part of quetiapine's antidepressant effect may be achieved through its restoration of sleep architecture.

\section{Methods}

\section{Study design}

This was a four-week, single-blind, open-label, repeated measures PSG study of patients with MDD or bipolar disorder currently experiencing a major depressive episode, who received quetiapine fumarate treatment in addition to their current medication regime. Patients were recruited from a single-center, tertiary care mood disorders clinic. The study was approved by the Queen's University Health Sciences and Affiliated Teaching Hospitals Research Ethics Boards as well as by the Health Canada Therapeutics Products Directorate.

\section{Study population}

Patients were recruited from inpatient and outpatient services for the evaluation and treatment of depression. All participants were 18 years of age or older and gave written informed consent. All patients had a Diagnostic and Statistical Manual of Mental Disorders, 4th Edition (DSM-IV) diagnosis of bipolar disorder (Type 1, Type 2, or not otherwise specified) or MDD, and were currently experiencing a major depressive episode (Hamilton Depression Rating Scale-17 item [HDRS-17] $\geq 15$, Young Mania Rating Scale [YMRS] $\leq 8$ ). Participants with a previous diagnosis of schizophrenia and/or current substance abuse (except caffeine and nicotine) were excluded. Prior antipsychotic use including quetiapine was allowed, but antipsychotics had to have been discontinued at least one week prior to study initiation. Sleep aids, including over-the-counter hypnotics, were discontinued at least one week prior to study initiation, and changes to baseline medications were not allowed in the three weeks prior to participation or during the study. Study participants were withdrawn from the study if they experienced significant side effects or withdrew consent.

Fifteen patients were enrolled in the trial. Four patients voluntarily withdrew from the study before completing a post-baseline PSG, two citing excessive sedation, one a post-menopausal bleed, and one a headache, and thus were not included in the completer analysis population. Therefore, only 11 patients were included in the analysis.

The completer population consisted of nine females and two males, with a mean age of $44.3( \pm 9.1)$ years. Five patients had a diagnosis of MDD and six patients had a diagnosis of bipolar disorder. Ten of 11 patients were taking antidepressants (five venlafaxine, one sertraline, two bupropion, one citalopram, and one a combination of bupropion and venlafaxine). Two patients were taking lithium and topiramate as mood stabilizers. Five patients possibly took sedating medications during the trial; three were taking established regimens of regular benzodiazepines prescribed as anxiolytics (one lorazepam, one clonazepam, and one a combination of oxazepam and lorazepam) and two were taking regular trazodone prescribed as an antidepressant. One patient had 
occasional sleep difficulties surrounding nocturnal priapism and another patient had obstructive sleep apnea and was being treated with continuous positive airway pressure.

\section{Quetiapine treatment}

Quetiapine was given in tablet form and taken at night near bedtime. An initial dose of $50 \mathrm{mg}$ was given and the dose was titrated up over the course of the study, with drug tolerance as the primary influence on titration. The average dose of quetiapine achieved was $155 \mathrm{mg}$, ranging from 100-200 mg. A fourth phase of the study, involving further titration of quetiapine to a target dose of $600 \mathrm{mg}$ per day and PSG, PSQI, and mood scales at days 42-48 was optional, and is not included here due to poor participation (five of 11 patients of the completer group).

\section{Clinical assessments}

Patients were assessed at three time points, ie, baseline (before administration of study medication), and after $2-4$ days and 21-28 days of quetiapine treatment. Each clinical assessment consisted of the HDRS-17, Montgomery Asberg Depression Rating Scale (MADRS), YMRS, Clinical Global Impression Severity (CGI-S) scale, and participant-reported PSQI.

\section{Polysomnography}

Objective sleep architecture measurements were derived from PSG data of study participants at defined intervals throughout the study period. A baseline PSG was taken on the day before quetiapine administration began, then once during days 2-4, and once during days 21-28. On each of the three study nights, sleep PSGs were set up by a qualified technician and recorded using the MediPalm Personal Recording Device (Braebon Corp., Ogdensburg, NY) while the patient slept at home. Patients were asked to retire and rise at their usual time. The standard overnight PSG sleep study included four electroencephalogram channels (C4-A1, C3-A2, O2-A1, O1-A2), electro-oculogram (two channels), submental electromyogram (EMG), pulse oximetry, oronasal airflow (oronasal thermistor), chest and abdominal movement (respiratory inductance plethysmography), and tibialis anterior EMG. A position sensor was used to monitor position continuously (Ultima Body Position Sensor; Braebon Corp., Ogdensburg, NY). The overnight sleep routine was applied starting at around 1900 hours each study night. Recording began at approximately 2100 hours, and ran for eight hours continuously or until the participant rose in the morning. One of two certified PSG analysts, different from the technician setting up the PSG and blinded to study design and day of study, scored the sleep record in 30-second segments according to the standardized criteria of Rechtschaffen and Kales, ${ }^{25}$ using Pursuit Advanced Sleep System software (Braebon Corp., Ogdensburg, NY).

Sleep onset was defined as the beginning of the first two minutes that were not scored as awake or movement. Latencies to each sleep stage were calculated to the first two continuous minutes of the stage. Obstructive apneas and hypopneas were scored using the criteria from the American Academy of Sleep Medicine Task Force. ${ }^{26}$ Events were scored when a $>50 \%$ decrease (apnea) in airflow, or clear reduction (hypopnea) in amplitude of the airflow signal (compared with stable breathing during the two minutes preceding the event), occurred associated with an arousal, a greater than $3 \%$ reduction in oxygen saturation $\left(\mathrm{SaO}_{2}\right)$, or both, and the event lasted for at least 10 seconds. Arousals were scored based on American Sleep Disorders Association criteria. ${ }^{27}$ Arousals had to be preceded by at least 10 seconds of sleep, have an electroencephalogram frequency shift to alpha or theta for at least three seconds and up to 15 seconds, and be associated with concurrent increased EMG tone in REM sleep. The respiratory disturbance index (RDI), which included apneas, hypopneas, and snore arousals for the number of events per hour of sleep, was calculated. Sleep efficiency (percentage) was calculated as the total sleep time divided by the total time in bed, multiplied by 100 .

\section{Statistical analysis}

The completer analysis population was comprised of the patients who had initiated quetiapine during the trial and had both a baseline and at least one post-baseline PSG measurement. Calculation of the sample size was based on choosing sleep efficiency as the primary outcome measure and estimating that a $15 \%$ improvement in sleep efficiency would be a clinically meaningful finding in this population. In the power calculation, we have used baseline sleep efficiency for depressed population to be 67.4 with a standard deviation (SD) $\pm 18.88 .{ }^{28}$ In order to detect a $15 \%$ improvement in sleep efficiency using one-sided normal distribution paired t-test analysis with a significance of 0.05 and $80 \%$ power, a total of 12 (11.277) patients is required. A sample size of 15 patients was used to allow for patient drop out.

Statistical analysis of data was done using the SPSS 17.0.1 for PC (SPSS Inc, Chicago, IL). Nonparametric analysis using the Wilcoxon Signed Rank test for two related samples, comparing pre- and post-treatment measures, was used because sample sizes were small and often not normally distributed (determined by Kolmogorov-Smirnov analysis). In those 
few pairwise comparisons in which both sets of data were normally distributed, additional analysis using two-sided normal distribution paired t-test analysis with a significance of 5\% was undertaken. Three of the 11 patients missed their day 21-28 PSG, one of whom had values substituted from their day 42-48 PSG administered during the optional extension period. An attempt to use multiple regression analysis to approximate missed PSG data failed to generate values consistent with prior observed values and, thus, for t-tests, the last observation was carried forward and substituted for missing values.

\section{Results \\ Sleep efficiency}

Quetiapine did not significantly alter sleep efficiency. Mean sleep efficiency ( \pm SD) was $69.8 \%$ ( \pm 20.6$), 78.4 \%$ $( \pm 9.7)$, and $74.5 \%( \pm 17.4)$ at baseline, and days $2-4$, and days $21-28$, respectively $(P=0.48$ and $P=0.21$, Figure 1 , Table 1).

\section{Sleep continuity}

No significant changes in total sleep time $(P=0.18)$, number of awakenings $(P=0.72)$, time in bed $(P=0.66)$, and latency to sleep onset $(P=0.51)$ were identified after initiation of quetiapine. A significant fall in the RDI was observed after 2-4 days of quetiapine treatment compared with baseline $(P=0.041)$. However, RDI then increased and there was no significant difference in RDI after 21-28 days of quetiapine treatment compared with pretreatment initiation $(P=0.86$, Table 1).

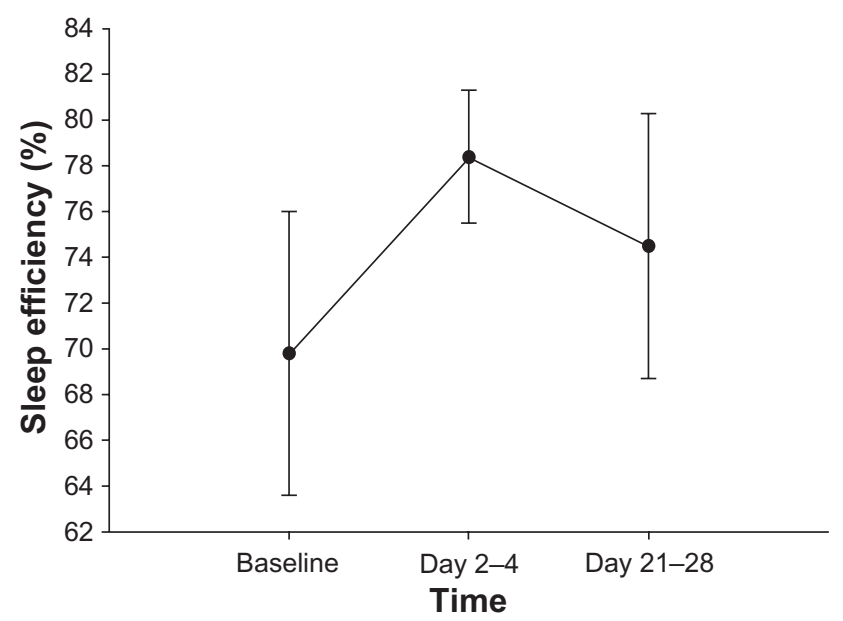

Figure I Mean \pm standard error of sleep efficiency (percentage) over one month of adjunctive quetiapine treatment in II subjects. Sleep was assessed by polysomnography at baseline, and after 2-4 days and 2I-28 days following initiation of quetiapine treatment. There was no significant difference in sleep efficiency after 2-4 days or one month of quetiapine treatment.

\section{Sleep architecture Non-REM sleep}

Acute quetiapine treatment significantly altered non-REM sleep stage characteristics, but longer-term treatment did not. The duration of both total Stage 2 sleep $(P=0.016)$ and total non-REM sleep $(P=0.05)$, as well as the percentage of total sleep time in non-REM sleep $(P=0.033)$ significantly increased 2-4 days after treatment initiation, compared with baseline, and then decreased towards baseline values. However, no significant difference in the above sleep measures was found between baseline and 21-28 days after treatment initiation (Table 1).

After 2-4 days of quetiapine treatment, total time in Stage 1 sleep $(P=0.16)$, percentage of total sleep time in Stage 1 sleep $(P=0.86)$, percentage of total sleep time in Stage 2 sleep $(P=0.06)$, percentage of total sleep time in SWS $(P=0.11)$, and total time in SWS $(P=0.20)$ were not significantly altered. After 3-4 weeks of treatment, percentage of total sleep time in Stage 1 sleep $(P=0.86)$, percentage of total sleep time in Stage 2 sleep $(P=0.95)$, total time in Stage 2 sleep $(P=0.86)$, percentage of total sleep time in non-REM sleep $(P=0.44)$, total time in non-REM sleep $(P=0.89)$, total time in SWS $(P=0.48)$, and percentage of total sleep time in SWS $(P=1.0)$ were not significantly altered compared with baseline measurements (Table 1).

\section{REM sleep}

Quetiapine initially altered REM sleep stage characteristics, but no significant changes from pretreatment measurements were observed after 21-28 days. The percentage of total sleep time in REM sleep significantly decreased 2-4 days after quetiapine treatment initiation $(P=0.033)$, although there was no significant difference in percentage of total sleep time in REM sleep between pretreatment and 3-4 weeks post-treatment ( $P=0.49$, Figure 1, Table 2$)$. After 2-4 days of treatment, there was no significant difference in latency to REM sleep $(P=0.86)$ and total time in REM sleep $(P=0.18)$ compared with pretreatment measurements. Three to four weeks of quetiapine treatment did not significantly alter latency to REM sleep $(P=0.59)$, percentage of total sleep time in REM sleep $(P=0.49)$, and total time in REM sleep $(P=0.49$, Table 1$)$.

\section{Subjective sleep quality}

PSQI scores did not significantly change between baseline, 2-4 days, and 21-28 days after initiation of quetiapine treatment, although PSQI scores tended to decrease over time ( $\mathrm{P}>0.05)$. After 21-28 days of quetiapine treatment, the 
Table I Sleep continuity and sleep architecture measures over one month of adjunctive quetiapine treatment in II subjects

\begin{tabular}{|c|c|c|c|c|c|}
\hline \multirow[t]{2}{*}{ Sleep parameter } & \multirow{2}{*}{$\frac{\text { Baseline }}{\text { Mean } \pm \text { SD }}$} & \multicolumn{2}{|l|}{ Days 2-4 } & \multicolumn{2}{|l|}{ Days 2I-28 } \\
\hline & & Mean \pm SD & $P$ value & Mean \pm SD & $P$ value \\
\hline \multicolumn{6}{|l|}{ Sleep continuity measure } \\
\hline Time in bed (minutes) & $479.4 \pm 33.9$ & $471.4 \pm 55.1$ & 0.657 & $478.7 \pm 32.3$ & 0.953 \\
\hline Total sleep time (minutes) & $332.9 \pm 91.0$ & $369.7 \pm 63.8$ & 0.182 & $353.3 \pm 70.6$ & 0.859 \\
\hline Sleep efficiency (\%) & $69.8 \pm 20.6$ & $78.4 \pm 9.7$ & 0.477 & $74.5 \pm 17.4$ & 0.213 \\
\hline Sleep onset latency (minutes) & $67.1 \pm 61.5$ & $57.1 \pm 51.7$ & 0.508 & $77.8 \pm 78.9$ & 0.515 \\
\hline Number of awakenings & $20.6 \pm 13.1$ & $23.6 \pm 16.0$ & 0.722 & $22.4 \pm 14.1$ & 0.833 \\
\hline RDI & $15.9 \pm 17.9$ & $12.6 \pm 17.9^{*}$ & $0.04 I$ & $15.5 \pm 16.2$ & 0.859 \\
\hline \multicolumn{6}{|l|}{ Non-REM measure } \\
\hline Stage I\% TST & $11.3 \pm 7.9$ & $11.3 \pm 6.7$ & 0.859 & $11.3 \pm 7.9$ & 0.859 \\
\hline Stage $2 \%$ TST & $54.9 \pm 14.4$ & $65.8 \pm 10.3$ & 0.062 & $60.0 \pm 10.2$ & 0.953 \\
\hline SWS\% TST & $12.1 \pm 9.9$ & $9.3 \pm 11.4$ & 0.114 & $12.2 \pm \mid 1.1$ & 1.000 \\
\hline Total non-REM\% TST & $78.3 \pm 17.5$ & $86.3 \pm 7.2^{*}$ & 0.033 & $83.5 \pm 9.4$ & $0.44 I$ \\
\hline Stage I duration (min) & $37.4 \pm 26.2$ & $42.2 \pm 25.6$ & 0.155 & $36.8 \pm 20.4$ & 0.515 \\
\hline Stage 2 duration (min) & $190.3 \pm 68.1$ & $244.4 \pm 62.1^{*}$ & 0.016 & $213.8 \pm 61.3$ & 0.859 \\
\hline SWS duration (min) & $42.9 \pm 42.5$ & $33.2 \pm 39.9$ & 0.202 & $41.9 \pm 39.3$ & 0.484 \\
\hline Non-REM duration (min) & $270.6 \pm 91.1$ & $319.8 \pm 62.5^{*}$ & 0.050 & $292.5 \pm 54.1$ & 0.889 \\
\hline \multicolumn{6}{|l|}{ REM measure } \\
\hline REM latency (minutes) & $226.1 \pm 111.7$ & $241.5 \pm 136.1$ & 0.859 & $196.9 \pm 112.5$ & 0.594 \\
\hline REM\% TST & $21.7 \pm 17.5$ & $13.7 \pm 7.2^{*}$ & 0.033 & $16.5 \pm 9.4$ & 0.491 \\
\hline REM duration (minutes) & $62.4 \pm 33.7$ & $49.9 \pm 29.4$ & 0.182 & $60.8 \pm 37.1$ & 0.494 \\
\hline
\end{tabular}

Values are mean and standard deviations. Sleep architecture was assessed by polysomnography at baseline, and at $2-4$ days and $21-28$ days postinitiation of quetiapine treatment. $* P \leq 0.05$ compared with baseline.

Abbreviations: RDI, respiratory disturbance index; TST, total sleep time; SWS, slow wave sleep; REM, rapid eye movement.

subjective sleep quality subscore of the PSQI was significantly lower than before treatment $(P<0.05)$, indicating that quetiapine significantly improved subjective sleep quality after four weeks. However, there was no significant difference in subjective sleep latency, sleep duration, habitual sleep efficiency, sleep disturbances, and sleep dysfunction subscores after 2-4 days or 21-28 days of treatment compared with baseline, although scores declined with the exception of habitual sleep efficiency, which remained unchanged (Table 2).

Table 2 PSQI scores over one month of quetiapine adjunctive treatment in II subjects

\begin{tabular}{llll}
\hline $\begin{array}{l}\text { PSQI sleep } \\
\text { questionnaire }\end{array}$ & $\begin{array}{l}\text { Baseline } \\
\text { (Mean } \pm \text { SD) }\end{array}$ & $\begin{array}{l}\text { Days 2-4 } \\
(\text { Mean } \pm \text { SD) }\end{array}$ & $\begin{array}{l}\text { Days 2I-28 } \\
\text { (Mean } \pm \text { SD) }\end{array}$ \\
\hline Subjective sleep quality & $2.5 \pm 0.5$ & $1.9 \pm 1.0$ & $1.3 \pm 0.9^{*}$ \\
Sleep latency & $2.0 \pm 1.2$ & $1.5 \pm 1.1$ & $1.4 \pm 1.2$ \\
Sleep duration & $1.2 \pm 1.0$ & $1.0 \pm 1.1$ & $0.5 \pm 0.8$ \\
Habitual sleep efficiency & $1.9 \pm 1.4$ & $1.0 \pm 1.2$ & $0.6 \pm 1.0$ \\
Sleep disturbances & $1.9 \pm 0.6$ & $1.5 \pm 0.5$ & $1.6 \pm 0.8$ \\
Use of sleep medication & $1.2 \pm 1.4$ & $1.0 \pm 1.3$ & $1.2 \pm 1.5$ \\
Daytime dysfunction & $2.2 \pm 0.6$ & $1.8 \pm 0.6$ & $1.7 \pm 0.9$ \\
Total score & $\mathbf{I 2 . 9} \pm \mathbf{4 . 1}$ & $\mathbf{9 . 7} \pm \mathbf{4 . 2}$ & $\mathbf{8 . 4} \pm \mathbf{4 . 9}$ \\
\hline
\end{tabular}

Mean score \pm SD of each item and total score on PSQI subjective sleep scale. PSQI scale was completed by participants at baseline, and after $2-4$ days and $2 I-28$ days of quetiapine treatment. $* P<0.05$ compared with baseline.

Abbreviations: PSQI, Pittsburg Sleep Quality Index; SD, standard deviation.

\section{Mood}

Quetiapine significantly improved mood and decreased depressive symptoms. Total MADRS scores significantly declined from baseline to days $2-4(P=0.01)$ and to days 21-28 $(P=0.008)$ of quetiapine treatment, to mean scores $( \pm \mathrm{SD})$ of $28.1( \pm 7.0), 22.4( \pm 7.9)$, and $14.1( \pm 8.4)$, respectively (Figure 2). Both HDRS and CGI-S total scores significantly decreased $(P=0.01$ and $P=0.02)$ from baseline mean scores $( \pm \mathrm{SD})$ of $22.3( \pm 4.4)$ and $4.5( \pm 1.2)$, respectively, to mean scores of $12.0( \pm 5.8)$ and $3.2( \pm 1.7)$ after 21-28 days of quetiapine treatment (Figures 3 and 4). YMRS mania scores remained low and statistically unchanged over time.

\section{Discussion}

This study demonstrates that adjunctive quetiapine treatment improves self-reported sleep quality, decreases illness severity, and initially alters sleep architecture in depressed patients. Alterations in sleep architecture are no longer seen after a longer treatment span. Specifically, quetiapine significantly increased total Stage 2 sleep time and total time spent in non-REM sleep, as well as decreased the percentage of total sleep time in REM sleep and the RDI after 2-4 days of treatment. However, these significant effects were not seen after 21-28 days of treatment. Depression significantly improved after 3-4 weeks of treatment. Therefore, it is evident that quetiapine adjunctive treatment in 


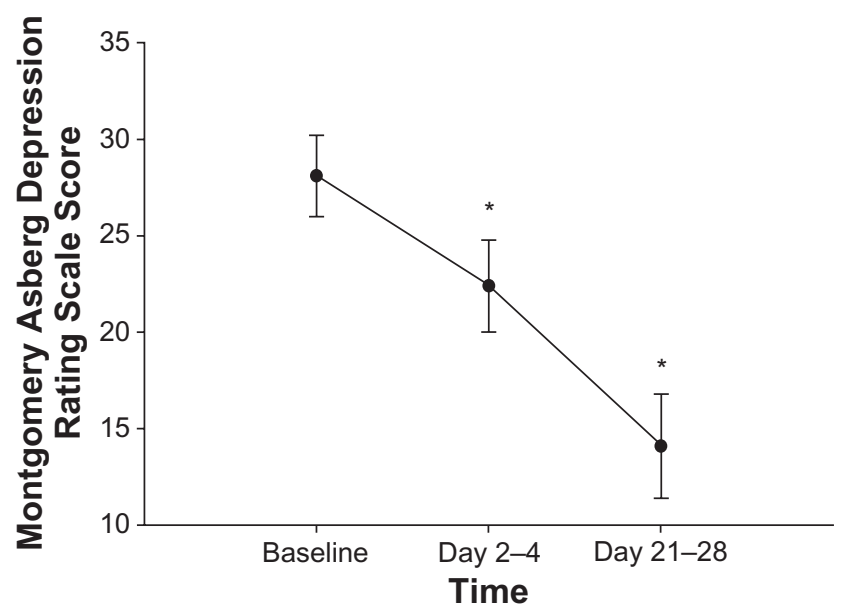

Figure 2 Mean \pm standard error of the MADRS score over one month of adjunctive quetiapine treatment in II subjects. MADRS score was assessed at baseline, after acute treatment ( $2-4$ days), and after longer-term treatment ( $2 \mathrm{I}-28$ days) with quetiapine. After 2-4 days and 2I-28 days of quetiapine treatment, MADRS scores significantly decreased from baseline measurements $(P=0.0 \mathrm{I}$ and $P=0.008$, respectively). $* P<0.05$ compared with baseline.

Abbreviation: MADRS, Montgomery Asperg Depression Rating Scale.

depressed patients induces changes in sleep architecture that are more robust and significant upon acute treatment and tend to taper off, approaching pretreatment values after 3-4 weeks of treatment. Mood continues to improve over time, with illness severity lowest after 3-4 weeks of treatment. These results emphasize that quetiapine relieves depressive symptoms effectively and rapidly and produces mild sleep benefits, with the exception of slightly increasing the number of awakenings and decreasing the latency to REM sleep.

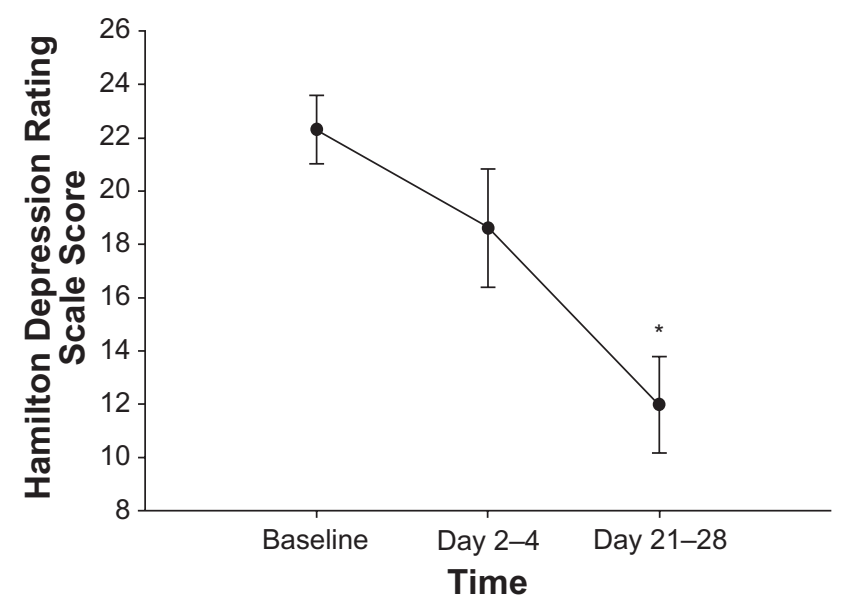

Figure 3 Mean \pm standard error of the HDRS score over one month of quetiapine adjunctive treatment in II subjects. HDRS-I7 score was assessed at baseline, after acute treatment ( $2-4$ days), and after longer-term treatment ( $2 \mathrm{I}-28$ days) with quetiapine. After $2 \mathrm{I}-28$ days of quetiapine treatment, HDRS scores significantly decreased from baseline measurements $(P=0.01)$. ${ }^{*} P<0.05$ compared with baseline.

Abbreviation: Hamilton Rating Scale for Depression.

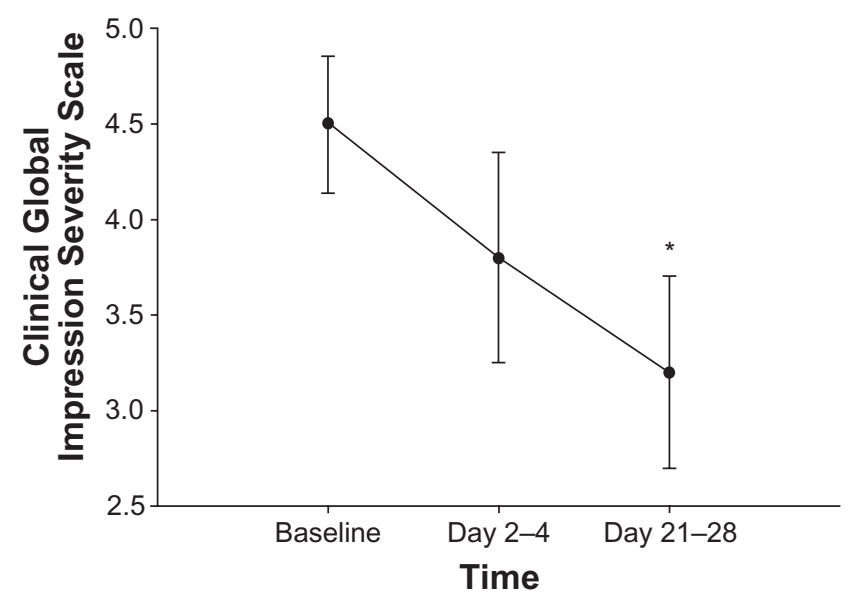

Figure 4 Mean \pm standard error of the CGI-S score over one month of quetiapine adjunctive treatment in II subjects. CGI-S score was assessed at baseline, after acute treatment ( $2-4$ days) and after longer-term treatment ( 2 I-28 days) with quetiapine. After 2I-28 days of quetiapine treatment, CGI-S scores significantly decreased from baseline measurements $(P=0.02)$. $* P<0.05$ compared with baseline.

Abbreviation: CGI-S, Clinical Global Impression Severity Scale.

Improvements in sleep continuity following quetiapine treatment have been observed in individuals who are not depressed. ${ }^{21,29}$ On the first night of treatment, Cohrs et al found that quetiapine significantly increased sleep period time, total sleep time, and sleep efficiency in healthy individuals. ${ }^{29}$ Moreover, in an open-label trial of patients with insomnia, total sleep time and sleep efficiency measured by PSG increased, and this increase extended over weeks of quetiapine treatment. ${ }^{21} \mathrm{To}$ our knowledge, the current study is the first to investigate sleep architecture, as well as observe changes in sleep in depressed patients undergoing quetiapine treatment, and in particular, patients who are resistant to SSRI treatment. It is important to note that pretreatment sleep efficiency values, with a mean of $69.8 \pm 20.6 \%$, in this study are comparable with those of other PSG studies in depressed patients $(67.4 \pm 18.9 \%),{ }^{28}$ indicating that there was no selection bias regarding the baseline sleep quality of participants in this study.

The effects of atypical antipsychotics on sleep architecture in depressed patients are not well documented. However, similar improvements in sleep following quetiapine treatment are also observed in the few other antipsychotics that have been studied. Adjunctive olanzapine treatment improves sleep continuity as well as increases percentage of sleep time in both non-REM sleep and SWS sleep in treatment-resistant patients with MDD. ${ }^{30}$ Risperidone decreases REM sleep and increases Stage 2 sleep in SSRI-resistant depressed patients. ${ }^{31}$ This study shows that quetiapine increases non-REM sleep, as does olanzapine, in addition to increasing Stage 2 sleep and decreasing REM sleep, as does risperidone. Quetiapine appears to alter sleep architecture initially in a manner similar to other 
medications in its class; however, unlike treatment with other antipsychotics, these improvements in sleep do not last.

Of particular interest is the finding that quetiapine suppressed REM sleep, as identified by decreased percentage of REM sleep. Depression is associated with REM sleep abnormalities including decreased REM latency, increased REM density, and increased REM time. ${ }^{32,33}$ It is possible that an initial decrease in REM sleep may be related to acute improvements in mood following quetiapine treatment; however, longer-term improvements in depressive symptomatology are not related to changes in sleep.

The sleep-inducing properties of quetiapine are most likely related to the medication's receptor-binding profile. Quetiapine is an antagonist at $\mathrm{H}_{1}$ receptors, inducing sleep upon inhibition of histamine synthesis. Histaminergic activity has more recently been implicated in the development of depression. Patients with depression show a decrease in $\mathrm{H}_{1}$ receptor binding, and this decrease is correlated with illness severity. ${ }^{34}$ Therefore, the modulation of histaminergic activity by quetiapine to induce sleep may be related to the reduction of depressive symptoms in these patients.

The most substantial changes in sleep occurred after 2-4 days of treatment, after which these changes began to decline, yet patients experienced the greatest improvement in mood after 21-28 days of quetiapine treatment. This leads us to conclude that, while psychotropic medications may act to reduce depressive symptoms by improving the sleep of patients with depression or bipolar disorder, sleep improvements are only a part of the medication's therapeutic action. Improvements in sleep contribute to a therapeutic response, but do not account on their own for the reduction in depressive symptomatology seen after quetiapine treatment.

The main limitations in this study are its open-label design, small sample size, lack of a control group, and a study population of predominantly women. Furthermore, sleep studies such as this one, acquire PSG data at distinct time points which may not be representative of the entire week. This study should be repeated as a randomized, placebo-controlled, double-blind assessment study with a larger sample size.

\section{Conclusion}

Acute quetiapine adjunctive therapy alters sleep architecture in depressed patients, after which these changes taper off towards baseline levels and are not significantly present after longerterm treatment. This pilot study suggests that further investigation of the effect of quetiapine on sleep architecture is warranted in patients experiencing a major depressive episode.

\section{Acknowledgments}

The authors would like to thank Alan Lowe, Regina du Toit, Judy Joannette, Teresa Garrah, Liane Tackaberry, Ann Shea and Dianne Groll.

\section{Disclosure}

This project was supported by an unrestricted research initiation grant from AstraZeneca.

LG, LL, DM and RJ declare no conflict of interest.

$\mathrm{RM}$ is on Speaker/Advisory Boards for, or has received research funds from: AstraZeneca, Biovail, BrainCells Inc., Canadian Network for Mood \& Anxiety Treatments, Eli Lilly, Janssen-Ortho, Lundbeck, Pfizer, Servier, Takeda, Wyeth, Bristol-Myers Squibb and Merck.

\section{References}

1. Nemeroff CB, Kinkhead B, Goldstein J. Quetiapine: Preclinical studies, pharmacokinetics, drug-interactions, and dosing. J Clin Psychiatry. 2002;63:5-11.

2. Calabrese JR, Keck PE, Macfadden W, et al. A randomized, doubleblind, placebo-controlled trial of quetiapine in the treatment of bipolar I or II depression. Am J Psychiatry. 2005;162:1351-1360.

3. Thase ME, Macfadden W, Weisler RH, et al. Efficacy of quetiapine monotherapy in bipolar I and II depression: A double-blind, placebocontrolled study (the BOLDER II study). J Clin Psychopharm. 2006;26:600-606.

4. Yatham LN, Kennedy SH, O'Donovan C, et al. Canadian network for mood and anxiety treatments (CANMAT) guidelines for the management of patients with bipolar disorder: Update 2007. Bipolar Disord. 2006;8:721-739.

5. Tohen M, Vieta E, Calabrese J, et al. Efficacy of olanzapine and olanzapine/fluoxetine combination in the treatment of bipolar I depression. Arch Gen Psychiatry. 2003;60:1079-1088.

6. Corya SA, Williamson D, Sanger TM, et al. A randomized, doubleblind comparison of olanzapine/fluoxetine combination, olanzapine, fluoxetine, and venlafaxine in treatment-resistant depression. Depress Anxiety. 2006;1:1-9.

7. Pathak S, Johns ES, Kowatch RA. Adjunctive quetiapine for treatmentresistant adolescent major depressive disorder: A case series. J Child Adolesc Psychopharmacol. 2005;15:696-702.

8. Milev R, Abraham G, Zaheer J. Add-on quetiapine for bipolar depression: A 12-month open-label trial. Can J Psychiatry. 2006;51:523-530.

9. Cutler A, Montgomery S, Feifel D, Lazarus A, Astrom M, Brecher M. Extended release quetiapine fumarate monotherapy in major depressive disorder: a placebo- and duloxetine-controlled study. J Clin Psychiatry. 2009;70:526-539.

10. El-Khalili N, Joyce M, Atkinson S, et al. Extended-release quetiapine fumarate (quetiapine XR) as adjunctive therapy in major depressive disorder (MDD) in patients with an inadequate response to ongoing antidepressant treatment: A multicentre, randomized, double-blind, placebo-controlled study. Int J Neuropsychopharmacol. 2010;23:1-16.

11. Bauer M, Pretorius HW, Constant EL, Earley WR, Szamosi J, Brecher M. Extended-release quetiapine as adjunct to an antidepressant in patients with major depressive disorder: Results of a randomized, placebocontrolled, double-blind study. J Clin Psychiatry. 2009;70:540-549.

12. Diagnostic and Statistical Manual of Mental Disorders 4th Ed, Text Revision (DSM-IV-TR). Washington, DC: American Psychiatric Association; 2000.

13. Riemann D, Berger M, Voderholzer U. Sleep and depression results from psychobiological studies: An overview. Biol Psychol. 2001;57:67-103. 
14. Breslau N, Roth T, Rosenthal L, Andreski P. Sleep disturbance and psychiatric disorders: A longitudinal epidemiological study of young adults. Biol Psychiatry. 1996;39:411-418.

15. Ford DE, Cooper-Patrick L. Sleep disturbances and mood disorders: An epidemiologic perspective. Depress Anxiety. 2001;14:3-6.

16. Perlis M, Giles DE, Buysse DJ, Tu X, Kupfer DJ. Self-reported sleep disturbance as a prodromal symptom in recurrent depression. J Affect Disord. 1997;42:209-212.

17. Liu X, Buysse DJ. Sleep and youth suicidal behavior: A neglected field. Curr Opin Psychiatry. 2005;19:288-293.

18. Tsuno N, Besset A, Ritchie K. Sleep and depression. J Clin Psychiatry. 2005;66:1254-1269.

19. Thase ME. Depression and sleep: Pathophysiology and treatment. Dialogues Clin Neurosci. 2006;8:217-226.

20. Cohrs S, Rodenbeck A, Guan Z, et al. Sleep-promoting properties of quetiapine in healthy subjects. Psychopharmacology (Berl). 2004; 174:421-429.

21. Wieband MH, Landry F, Brückner T, et al. Quetiapine in primary insomnia: A pilot study. Psychopharmacology (Berl). 2008;196:337-338.

22. Linden M, Thiels C. Epidemiology of prescriptions for neuroleptic drugs: Tranquilizers rather than antipsychotics. Pharmacopsychiatry. 2001;34:150-154

23. Baune BT, Caliskan S, Todder D. Effects of adjunctive antidepressant therapy with quetiapine on clinical outcome, quality of sleep and daytime motor activity in patients with treatment-resistant depression. Hum Psychopharmacol. 2007;22:1-9.

24. Endicott J, Paulsson B, Gustafsson U, Schiöler H, Hassan M. Quetiapine monotherapy in the treatment of depressive episodes of bipolar I and II disorder: Improvements in quality of life and quality of sleep. $J$ Affect Disord. 2008;111:306-319.
25. Rechtschaffen A, Kales A. A Manual of Standardized Terminology, Techniques and Scoring System for Sleep Stages of Human Subjects. Bethesda, MD: National Institutes of Health; 1968.

26. Sleep-related breathing disorders in adults: Recommendations for syndrome definition and measurement techniques in clinical research. The Report of an American Academy of Sleep Medicine Task Force. Sleep. 1999;22:667-689.

27. EEG arousals: Scoring rules and examples: A preliminary report from the Sleep Disorders Atlas Task Force of the American Sleep Disorders Association. Sleep. 1992;15:173-184.

28. Kerkhofs M, Linkowski P, Lucas F, Mendelwicz J. Twenty-four hour patterns of sleep in depression. Sleep. 1991;14:501-506.

29. Cohrs S, Rodenbeck A, Guan Z, et al. Sleep-promoting properties of quetiapine in healthy subjects. Psychopharmacology (Berl). 2004; $174: 421-429$.

30. Sharpley AL, Attenburrow ME, Hafizi S, Cowen PJ. Olanzapine increases slow wave sleep and sleep continuity in SSRI-resistant depressed patients. J Clin Psychiatry. 2005;66:450-454.

31. Sharpley AL, Bhagwagar Z, Hafizi S, et al. Risperidone augmentation decreases rapid eye movement sleep and decreases wake in treatmentresistant depressed patients. J Clin Psychiatry. 2003;64:192-196.

32. Benca RM, Obermeyer WH, Thisted RA, Gillin JC. Sleep and psychiatric disorders: A meta-analysis. Arch Gen Psychiatry. 1992;49:651-668.

33. Benca R, Okawa M, Uchiyama M, et al. Sleep and mood disorders. Sleep Med Rev. 1997;1:45-56.

34. Kano M, Fukudo S, Tashiro A, et al. Decreased histamine H1 receptor binding in the brain of depressed patients. Eur J Neurosci. 2004;20:803-810
Neuropsychiatric Disease and Treatment

\section{Publish your work in this journal}

Neuropsychiatric Disease and Treatment is an international, peerreviewed journal of clinical therapeutics and pharmacology focusing on concise rapid reporting of clinical or pre-clinical studies on a range of neuropsychiatric and neurological disorders. This journal is indexed on PubMed Central, the 'PsycINFO' database and CAS, and is the official

\section{Dovepress}

journal of The International Neuropsychiatric Association (INA). The manuscript management system is completely online and includes a very quick and fair peer-review system, which is all easy to use. Visit http://www.dovepress.com/testimonials.php to read real quotes from published authors. 